\title{
EU-Lobbying - Wegweiser durch ein Labyrinth
}

\author{
Schendelen, Rinus van: Die Kunst des EU-Lobbyings. Erfolgreiches Public Affairs Management \\ im Labyrinth Brüssels, Lexxion Verlagsgesellschaft, Berlin 2012, 478 Seiten, € 39,90.
}

Public Affairs, vulgo Lobbyismus, ist auch nach dem Eindruck der Adressaten offenbar keine dröge Angelegenheit. Auf einer Verbandstagung der Abfallwirtschaft stichelte vor wenigen Jahren eine damals amtierende Umweltministerin aus dem Südwesten Deutschlands, sie habe Erfahrungen mit den intensiven Beeinflussungsanstrengungen durch Interessengruppen des Gesundheitswesens sammeln dürfen, als sie noch Sozialministerin war. Diese „Aufmerksamkeit“ werde indes locker vom Lobbyistenkampf in der Entsorgungswirtschaft überboten. Die Angesprochenen nahmen die Worte als versteckte Respektsbezeugung an. Jüngst ergänzte Bundesumweltminister Peter Altmaier (CDU) den Befund mit der Feststellung, beim Ringen der Interessenvertreter im Entsorgungssektor sei die Luft „bleihaltig“.

Doch bevor die Stahlgewitter niederprasseln können, bedarf es einer klugen und sorgfältigen Vorbereitung durch die Experten, die ihr Tun in der Regel als Public Affairs bezeichnen, von außen aber häufig Lobbyisten genannt werden. Mit seinem erstmals in deutscher Sprache erschienenen Werk „Die Kunst des EU-Lobbying“ legt Rinus van Schendelen, Professor für Politikwissenschaft an der Erasmus-Universität zu Rotterdam, für das Public-Affairs-Management „im Labyrinth Brüssels“ ein praxisorientiertes Vademecum mitsamt einer kenntnisreichen Beschreibung des institutionellen Brüsseler „Zielgebiets“vor. Das Buch hebt sich dadurch von der vorhandenen, oft organisationssoziologischen Literatur ab.

Die deutsche Fassung präsentiert sich gegenüber der letzten englischsprachigen Auflage ${ }^{1}$ deutlich erweitert. Auf den Machiavelli-Bezug im Originaltitel verzichtet der Verlag gegenüber den deutschsprachigen Lesern. Warum? Vermutlich, weil der vielseitige Florentiner, dessen zentrale Gedanken stets um kluge, zweckgerichtete Daseinsbewältigung kreisten, hierzulande vornehmlich als ruchloser Ratgeber einer ethikfreien Machtpolitik gilt. Van Schendelen jedenfalls erblickt im intelligenten, methodischen Vorgehen à la Machiavelliklare Zieldefinition, sorgfältige Aufklärung, überlegte Wahl der individuell einzusetzenden Techniken und Mittel - und weniger in den Faktoren Prestige, Status oder Reichtum der Lobbygruppe das primäre Erfolgsrezept.

Ein überlegtes Management der entscheidenden Einflussfaktoren erfordert besonders die von mehr als 20.000 Einflussnehmern bespielte und von einer Vielzahl politische und administrativer Akteure bevölkerten polyzentrischen Brüsseler Bühne, die nach einer älteren Einschätzung des Buchautors als „Champions League des Lobbying“2 gelten darf. Die Analyse des europäischen Spielfelds, das Spielen auf der Public-Affairs-Klaviatur, das AgendaSetting stehen daher im Mittelpunkt des Buches. Die Kapitel werden mit instruktiven Fallstudien angereichert zum Beispiel zum Kampf um die Zulassung gentechnisch veränderter Lebensmittel.

Doch van Schendelens Werk erschöpft sich nicht in handwerklichen Ratschlägen. Von strategischer Bedeutung sind die Auskünfte, dass „Brüssel“ jährlich rund 2.500 neue ver-

1 Rinus van Schendelen, More Machiavelli in Brussels. The Art of Lobbying the EU, Amsterdam 2010.

2 Ders., Brüssel: Die Champions League des Lobbying, in: Thomas Leif/ Rudolf Speth (Hrsg.), Die fünfte Gewalt. Lobbyismus in Deutschland, Bonn 2006, S. 132 - 162. 
bindliche Entscheidungen produziert, die auf die Gesetzgebung der Mitgliedstaaten durchschlagen. Dabei sind die europäischen Institutionen offen für Einflussnahme: zuvörderst das Parlament, gefolgt von der Kommission, am wenigsten hingegen der Rat. Die Kommission ruft bereits im Vorfeld eines Gesetzgebungsprozesses, bei der Konsultation, Wissen und Positionen von Stakeholdern ab und macht dadurch auch einen vom Autor konstatierten personellen Ressourcenmangel wett. Interessant ist auch die Beobachtung, dass Interessenvertreter, deren Anliegen in ihrem Mitgliedstaat kein Erfolg beschieden war, wirkungsvoll die EU-Ebene bearbeiten, um ihre Sache in ihrem Heimatland via Brüssel doch noch durchzusetzen.

Nicht nur Wirtschaftsverbände und größere Unternehmen sind in „Europas Hauptstadt“ präsent, sondern auch Gewerkschaften, der öffentliche Sektor, Umweltorganisationen sowie Kirchen und andere mehr oder weniger sinnstiftende Einrichtungen. Professionalisierungsgrad, Reichweite der jeweiligen Aktivität und handwerkliches Vermögen sind dabei stark unterschiedlich. Wenngleich der Autor keiner Gruppe Perfektion attestieren kann, hebt er doch die Qualität des Public-Affairs-Management komplexer multinationaler Organisationen hervor. Sie hätten nicht nur eine kulturelle Vielschichtigkeit widerzuspiegeln, sondern müssten das Brüsseler Spielfeld deutlich breiter bespielen als Ein-Punkt-Akteure.

Wenig bekümmert van Schendelen die in einer freiheitlichen Ordnung eher abseitig klingende Klage, Lobbyismus sei „eine Macht ohne Legitimation“3. Der Autor - sich ansonsten normativ zurückhaltend - vertritt nachgerade die Gegenthese: Lobbygruppen tragen zur EU-Demokratie bei. Van Schendelen sieht die EU als „ein in höchstem Maße offenes und konkurrierendes“, demokratisches Input-System, für das Lobbygruppen hilfreich, aber selbstverständlich nicht ausreichend sind. Ungleichgewichte zwischen Interessengruppen können durch handwerkliche Professionalisierung abgemildert werden. Außerdem öffnen sich in der Brüsseler Input-Demokratie häufig die Türen von innen. Gegen Versuche einzelner Lobbygruppen, sich einen exklusiven Zugang zu den Entscheidern zu sichern, hat sich der Brüsseler Apparat bislang als wohltuend resistent erwiesen.

In den letzten Jahren hat sich nicht nur auf dem Brüsseler Parkett ein Wandel vollzogen. Viele Wirtschaftsunternehmen nehmen ihre Interessen durch eigene Firmenvertreter wahr, die zusätzlich zu den etablierten und keineswegs entbehrlich gewordenen Verbänden agieren. Führt diese Entwicklung zum sinnlosen Overkill oder zur Erschöpfung der Zielpersonen? Ein führender Parlamentarier der SPD-Bundestagsfraktion hat sich bei der Vorstellung des besprochenen Buches in Berlin dazu geäußert. Er sehe die Nützlichkeit des Trends: Unternehmensvertreter lieferten ihm vielfach wertvollere Marktinformationen und konturiertere Positionen als Repräsentanten von Verbänden, die zwangsläufig bloß einen Minimalkonsens ihrer Mitglieder kommunizieren könnten.

Jost Vielhaber

3 Thomas Leif / Rudolf Speth, Zehn zusammenfassende Thesen zur Anatomie des Lobbyismus in Deutschland und sechs praktische Lösungsvorschläge zu seiner Demokratisierung, in: dies., a.a.O. (Fn. 2), S. 352. 